\title{
Operating System using CAN Bus on Different Process Circulated Technology with High Speed Prime Marine Engine
}

\author{
R.Sundar, Stephen Arputharaj
}

\begin{abstract}
The objective of this work is marine main diesel engine with four stroke and normal speed operating system depend on circulated dealing out and failure tolerant Control area topology control arrangement correspondence innovation was exhibited. It incorporates various units like motor control unit, demonstrating board unit, motor wellbeing unit, primary motor interface, engine control unit computerized representative unit and dispersed preparing unit. Framework information can be traded by double repetitive CAN organize. The appropriated handling unit is free in material science totally; it contains less units effect even if few units broke down. The correspondence of framework cell is composed by set of rules correspondence convention of CAN, which individual parameter analyze. The Unit has capacity of individual -protection when misfortune influence, and local unit decide to stop the point its abnormal in speed insufficient of air it interact the local unit and high priority interface and control the engine are advantageous and amicable.
\end{abstract}

\section{INTRODUCTION}

Present, propelled Engine Main distance operating System is interact by means of main engine propulsion governor unit designed by Kongsberg Auto Chief C20 centralized unit and the Auto Chief 4 Propulsion Control System (AC4) propulsion control system that can be adapted to various engines. which are used as a bit of wide range slow run and double stroke ancillary marine diesel engine used if necessary. In general $\mathrm{AC} 4$ is incorporates to less than the level of range AC C20 sofar. But the advancement of this to incorporated with this to advance unit like more than the this due to level of artificial intelligence robotization. The framework exhibited in this proposition is composed by the normal-speed marine driven associated with turning around apparatus as the reverse electrical actuator protest, it compare and retains the outlined focal points of AC C20. This proposition of these examinations is framework equipment circulation, practical structure and segment rule of Prime Engine governor operating system from the point of view of framework advancement [3].

\section{DESIGN OF STYSTEM CONFIGUARATION}

The Prime Engine distance governor unit arrangement in perspective of detached configuration (Figure 1), it has many modules involved expansion unit (deck) ,associate with local control unit and engine control room unit (ECR) . All framework information can be working on double

Revised Manuscript Received on July 18, 2019.

R.Sundar, Assistant Professor, Department of EEE, AMET Deemed to be UNIVERSITY Chennai,India. (sundar.r@ametuniv.ac.in)

Dr.Stephen Arputharaj, Professor, Department of EEE, AMET Deemed to be UNIVERSITY Chennai,India. (stephen_arputharaj@rediffmail.com) Propulsion Control System (AC C20) operation is

repetitive CAN Bus organize by open network protocol correspondence convention built up. It can spare and bolt the present information when framework misfortune influence or have blame. Every correspondence secluded has a different handling unit for intelligent operation. The CAN organize convention is outlined as per set up normal information trade convention [4]. Next to, quantities of info and yield interfaces in framework are set to control for a comparable protest for various working frameworks.

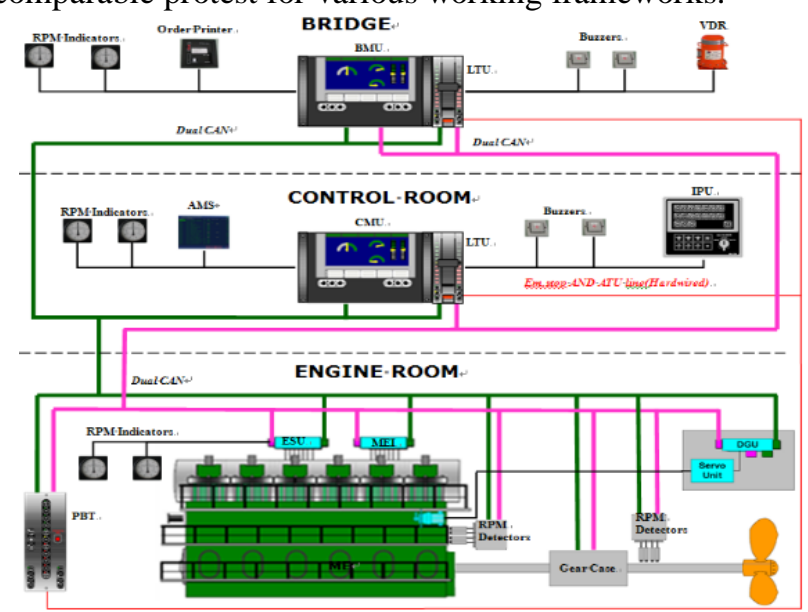

Figure 1: Prime Engine Controller configuration.

\section{CONFIGURATION OF VARIOUS MODULES}

\section{A. Configured of Command based changing station}

Working can be change over based on priority command requested to station and these can be implement by getting consent from low priority working station. The guidelines of working need to change over switch as taking based on the protocol after 1) The territory has the most indispensable ace; 2) Machinery control unit has higher priority than the deck .The Changeover can be refined by taking place of deck, machinery control unit and local. The Changeover between engine rooms to local unit on high priority basis based on the command given by the input signal [5]

\section{B. Operation of Speed mechanism Unit}

By the instruction of frame work of high priority instruction to implement by changing the operating station from distance control to local operating station is handled by changing set point "OFF" to "Toward the back" immediately the distance unit would set the machinery

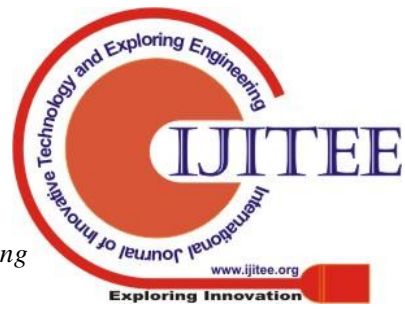




\section{OPERATING SYSTEM USING CAN BUS ON DIFFERENT PROCESS CIRCULATED TECHNOLOGY WITH HIGH SPEED PRIME MARINE ENGINE}

speed "clutch in " unintended changing is at lower than the higher interim for grip in. By this instant, the speed is not normalized to "Permit Clutch In Speed" at that point grip in persuasively. When the distance operating station is set from "Toward the back "to "off" point the distance operating station is initially allow to "grip out speed "Naturally and grip out exactly the higher interim for grasp out. Amid this duration, the speed is not normalized to "Permit Clutch Out speed",", at that point grasp out persuasively.

\section{DESIGN OF REGULATOR CONFIGURATION \& RESULTS}

Computerized senator regulator is the center of ME distance Control unit. Fig 2 demonstrates the computerized senator work square chart. The capacity configuration as depicted beneath[6]

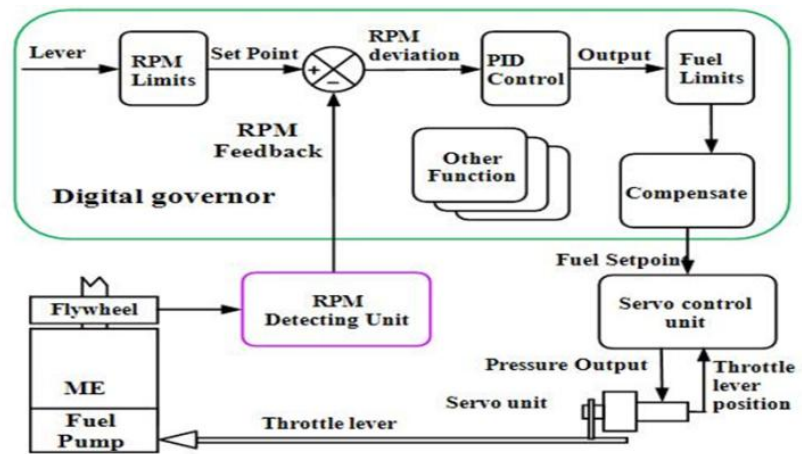

Figure 2: DESIGN OF REGULATOR CONFIGURATION

\section{A. Limitation of range of load and speed.}

To eliminate the friction losses influence to cause the decline in speed, heat exchange it may deteriorate the main engine running status, the Main engine governor unit set points are the aped and load range, it protects the breakthrough and maintains the more stable. This unit governs the various parameters like acceleration, velocity, temperature, loading, propulsion unit by manually to avoid the thrown off.
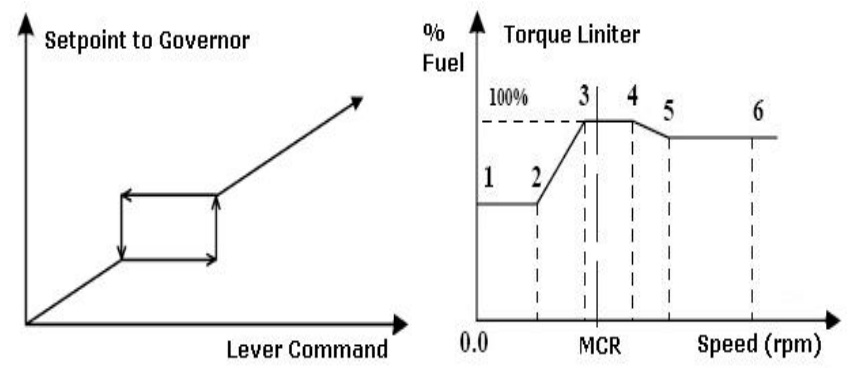

Figure 3: Breakthrough speed range and torque range model

\section{B. Consistent flow Fuel flow trend}

The main parameter influence to control the speed is fuel rate in which is regularly monitored and controlled by the set point. if the speed is remains constant the regulator the intimate the fuel mode is consistent. At that mode the regulator has not triggered the fuel control device to fixed mode. When the fuel valve is closed, the speed of the propeller may vary distortions as shown in figure 6 . At that interval the speed parameter is continuously measured and infer with the peak and off peak point. if the speed goes beyond the set point that is the peak and off peak point, the fuel valve is change to on mode thus the speed of the engine is controlled by the distance control unit. Thus the speed of the engine is continuously monitored and controlled by means of Consistent flow Fuel flow trend configuration method.This ensures that it would not allow the lower the negative peak not allowed to move to engine off mode.if the parameter goes overshoot the engine speed not shootup.thus it regulats.
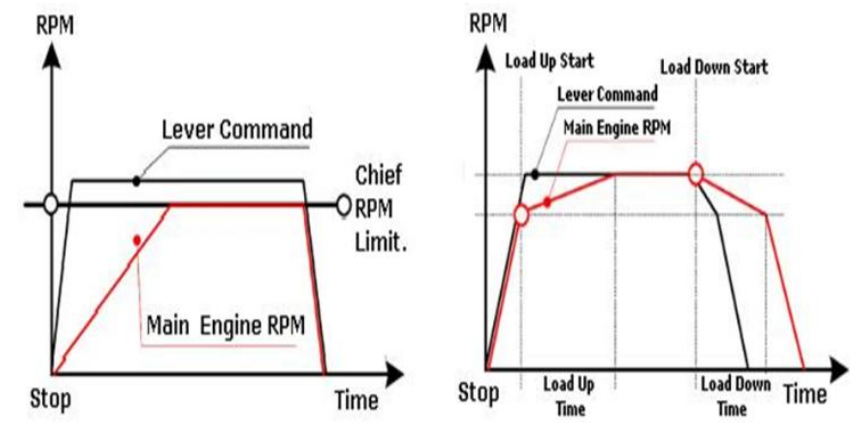

Figure 4: Constant fuel control trends

\section{CONCLUSION}

The Marine distance governing System is planned for focus speed and four-stroke marine diesel engine, which in light of passed on getting ready and twofold dreary CAN compose correspondence advancement. It consolidate structure essential diagram, task station switch setup, gearbox's grip in and grip out arrangement, propelled agent configured, speed estimation and analysis unit plot. Structure information can be exchanged by two fold redundant CAN organize [10]. The correspondence of structure units makes them check and researching limit. After implementation on ship , the execution of structure is fulfilled all kind of parameters are coincides the expectations of the essentials of usage in the field of marine.

\section{REFERENCES}

1. Cassez, F., Jard, C., Rozoy, B. and Ryan, M.D. eds., 2003. Modeling and Verification of Parallel Processes: 4th Summer School, MOVEP 2000, Nantes, France, June 19-23, 2000 Revised Tutorial Lectures (Vol. 2067). Springer.

2. Merz, S. and Cassez, F., 2001. Modeling and Verification of Parallel Processes. Springer-Verlag, LNCS, 2067, pp.3-38.

3. Dou, W.C., Xi, X.P. and Cai, S.J., 2002. Distributed Processing Oriented Workflow Modeling. COMPUTER INTEGRATED MANUFACTURING SYSTEMS-BEIJING-, 8(7), pp.533-537.

4. Fang, S.X. and Li, Q., 2006. Design and Application of a CAN Higher Layer Protocol with Master-Slave Mode (J). Measurement \& Control Technology, 25(08), pp.47-49.

5. Tang, X.J., Li, H.M., Jin, H.B., Yu, F.P., Peng, H.P. and Zhou, T.B., 2003. Development of Remote Control System for Middle Speed Marine Diesel Engine (J). Journal of Wuhan University of Technology, 27(05), pp.625-628.

6. Zhang, G.C., 2008. Development of Simulator on Digital Speed Regulator for Marine Main Engine (J). Ship \& Ocean Engineering, 37(05), pp.50-54. 
7. Zhang, G.C. and Ren, G., 2008. Research and Realization of PID+ Digital Speed Regulator for Main Engine (J). Navigation of China, 31(02), pp.117-121.

8. Meng, J. and Ma, J., 2009. Controlling Technology and Clutch Model of Twin-engine Incorporation (J). Ship Engineering, 31, pp.58-62.

9. Zheng, W.M. and Huang, Y.X., 2000. The Design and Analysis on Speed Feedback Link of Main Engine Remote Control System (J). Journal of Jimei University (Natural Science), 5(4), pp.7-10.

10. Jinchu, H.U., 2002. The design of Large Power Monitoring System Based on Distributed Process [J]. Computing Technology and Automation, 3, p.019.

11. Vettriselvan R., Ruben Anto., \& Jesu Rajan FSA (2018), Rural lighting for energy conservations and sustainable development, International Journal of Mechanical Engineering and Technology, 9(7):604-611.

12. Vettriselvan R., \& Ruben Anto., (2018) Pathetic Health Status and Working Condition of Zambian Women, Indian Journal of Public Health Research \& Development, 9(9):259-264.

13. Vettriselvan R., Sathya M., \& Velmurugan T. (2018), Productivity and Profitability Mechanical Engineering Entrepreneurs: Business Perspective, International Journal of Mechanical Engineering and Technology, 9(8): 758-765. 